\title{
LA FÍSICA, CIENCIA TEÓRICA Y EXPERIMENTAL
}

\section{PHYSICS, THEORETICAL AND EXPERIMENTAL SCIENCE}

Julio Gutiérrez Muñoz: Universidad de Alcalá de Henares. Madrid (España) julio.gutierrez@uah.es

\section{CURRÍCULUM VITAE}

Catedrático de Física Atómica, Molecular y Nuclear de la Escuela Politécnica Superior de la Universidad de Alcalá de Henares (Madrid). En la actualidad imparte clases de la asignatura de Física incluida en la titulación de Grado en Química de dicha Universidad. Autor de numerosos artículos científicos. Miembro fundador de GRUA (Grupo de Reflexión de la Universidad de Alcalá de Henares). Vicerrector de la Universidad de Alcalá de Henares. Director de la Revista Vivat Academia.

\section{RESUMEN}

La Ciencia, en sentido extenso, es el producto de la propia actividad humana, en su lucha por la supervivencia de la especie, siempre encaminada al hallazgo de objetos que mejoren las condiciones de vida y supervivencia. La Física representa la Ciencia en su estado más básico y en sus aspectos experimentales más fundamentales. El mundo físico es nuestro propio entorno, el conjunto de aquello que podemos aprehender con nuestros sentidos. El Hombre, en su natural curiosidad, siempre ha deseado conocer y dominar esas reglas por las que la Naturaleza se ha regido, se rige, y seguirá rigiéndose, en su evolución. Por ello, el papel de la Física es fundamental, porque estudia las leyes que rigen los fenómenos más básicos de la 
Naturaleza y la relación entre ellos. La Ciencia, en general, y la Física, en particular, han hecho posible una mejora apreciable de nuestra calidad y esperanza de vida.

\title{
PALABRAS CLAVE
}

Ciencia - Física - Hombre - Naturaleza - Supervivencia

\begin{abstract}
Science in the extended sense, is the product of human activity itself, in its struggle for survival of the species, always aimed at finding objects that improve living conditions and survival. The Physical Science is at its most basic and most fundamental experimental aspects. The physical world is our own environment, the set of what we can apprehend with our senses. Man in his natural curiosity, always wanted to know and master those rules by which nature has ruled, governed, and will continue to be governed, in its evolution. Therefore, the role of physics is essential, because it studies the laws governing the most basic phenomena of nature and the relationship between them. Science in general and physics in particular, have made possible a significant improvement in our quality and life expectancy.
\end{abstract}

\section{KEY WORDS}

Science - Physics - Men - Nature - Survival

\section{ÍNDICE}

1. Introducción

2. El mundo físico. Los modelos

3. Notas 


\section{TEXTO}

\section{Introducción}

Podemos definir la Ciencia en sentido laxo o en sentido restringido. En el primer supuesto, Ciencia sería el producto de la propia actividad humana, en su lucha por la supervivencia de la especie. Así, Ciencia es el descubrimiento del uso de objetos contundentes para abrir frutos sin romperse la dentadura, el lanzamiento de esos objetos para derribar presas o enemigos, el descubrimiento del fuego, de la alfarería, de la selección de especies en agricultura y ganadería, etc., etc. En estos casos, Ciencia es el conjunto de esfuerzos y hallazgos que forman parte de la memoria colectiva de la Humanidad, encaminados a mejorar sus condiciones de vida y supervivencia, debidamente estructurados, organizados e interrelacionados, a fin de poder transmitirlos de generación en generación, por no constituir parte de la herencia genética.

En un sentido más estricto, podemos definir Ciencia como el conjunto de conocimientos que describen el orden interno de la Naturaleza, sus causas y las leyes que gobiernan las relaciones existentes entre los diferentes fenómenos que podemos percibir.

En sus dos acepciones, la Ciencia nace con la propia conciencia del Hombre como especie diferente y curiosa, mucho antes de hacerse preguntas del porqué de su existencia, su origen o destino. En realidad, la religión como actividad intelectual, no es más que el intento de explicar muchos de esos fenómenos que escapan al análisis racional del individuo, por las propias limitaciones de las experiencias que puede realizar. Es en este sentido cuando la Ciencia cobra su verdadera dimensión experimental. No puede haber Ciencia sin análisis experimental, aunque sea en 
sentido figurado, haciendo uso de la capacidad humana de razonar para plantearse problemas reales o ficticios.

\section{El mundo físico. Los modelos}

Dentro de la actividad científica en general, la Física representa la Ciencia en su estado más básico y en sus aspectos experimentales más fundamentales. Analicemos con más detenimiento esta definición.

Cualquier suceso que tiene lugar ante nuestros ojos, identificado como un cambio de nuestro entorno a través de la información proporcionada por nuestros sentidos, ocurre obedeciendo una serie de reglas fundamentales -al menos así nos parece-, ajenas a nuestra voluntad, que pueden ser muy complicadas de entender, muy difíciles de descubrir o, por el contrario, muy simples de establecer: estos sucesos los conocemos por el nombre de fenómenos físicos. En una definición tan general, fenómenos físicos son todos, y el ámbito de actuación de la Física es la Naturaleza en su conjunto. En otras palabras, mundo físico es nuestro propio entorno, el conjunto de aquello que podemos aprehender con nuestros sentidos. Entonces, ¿qué papel les dejamos interpretar al resto de las Ciencias en el Teatro Natural? Resulta difícil contestar esta pregunta, pero lo intentaremos en los párrafos siguientes.

El Hombre, en su natural curiosidad, siempre ha deseado conocer y dominar esas reglas por las que la Naturaleza se ha regido, se rige, y seguirá rigiéndose, en su evolución. La mayoría de las veces, esas pautas, que se cumplen indefectiblemente, pueden ser establecidas, sin que sepamos su porqué, las llamamos entonces principios físicos. Otras veces, las reglas pueden derivarse de otras encontradas con anterioridad, aunque su primer análisis se haya realizado independientemente de estas últimas, y las llamaremos simplemente leyes físicas. NOTA_1 El conocimiento que los humanos, a lo largo de su evolución, han ido adquiriendo de estos principios 
y leyes y sus relaciones lo han plasmado en un cuerpo de doctrina conocida con el nombre de Física. Estas Leyes y Principios, establecidos con la ayuda de la experiencia, la Lógica y las Matemáticas, permiten hacer predicciones que nos ayudan a controlar nuestro entorno y, por tanto, han servido, a su vez, para que se hayan desarrollado, hasta el grado extraordinario que todos conocemos, la Ingeniería y la Tecnología, así como las demás Ciencias.

Recapitulemos. Cuando miramos a nuestro alrededor y observamos la gran cantidad de utensilios que están a nuestra disposición, cuyo funcionamiento desconocemos pero que usamos con toda naturalidad (televisión, horno microondas, radiadores de infrarrojos, relojes, ordenadores, automóviles, etc., etc), tenemos la impresión de estar inmersos en un mundo dominado por los avances científicos. En todos estos avances la Física ha tenido y tiene un papel fundamental, pues estudia las leyes que rigen los fenómenos más básicos de la Naturaleza y la relación entre ellos. Este estudio hace posible la predicción de nuevos fenómenos, comportamientos diferentes y, en definitiva, la utilización de esas leyes para nuestro provecho, haciéndonos más agradable la existencia. Al menos esa debe ser la intención primera del científico.
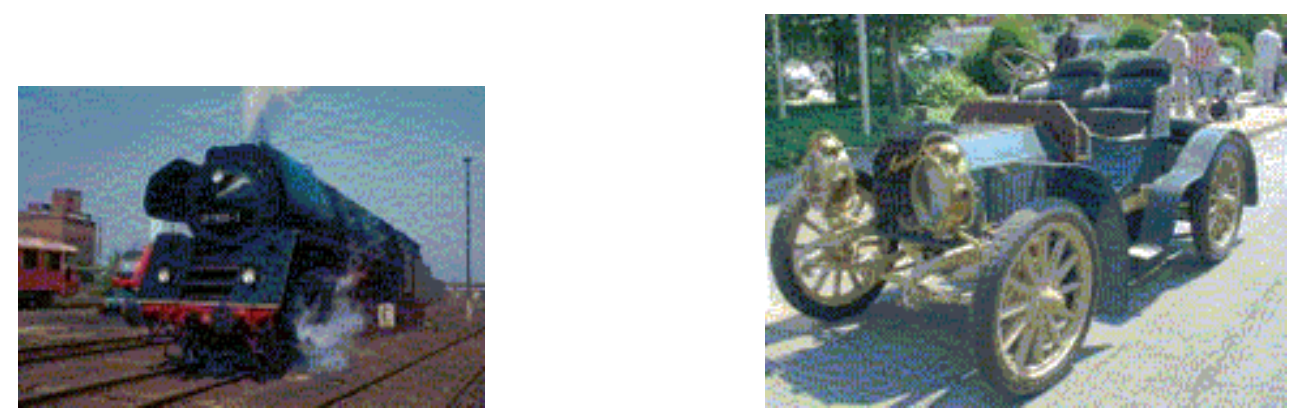

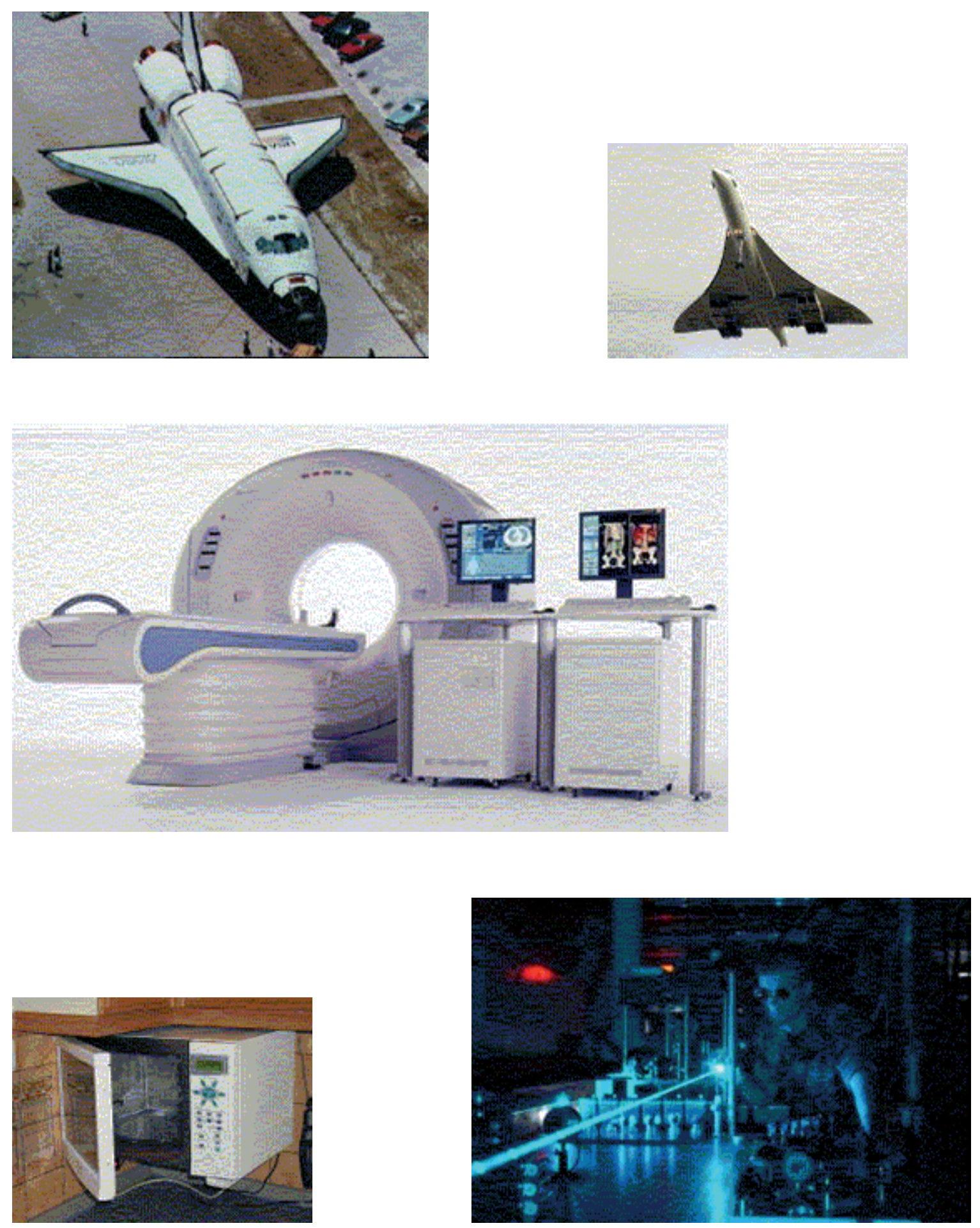

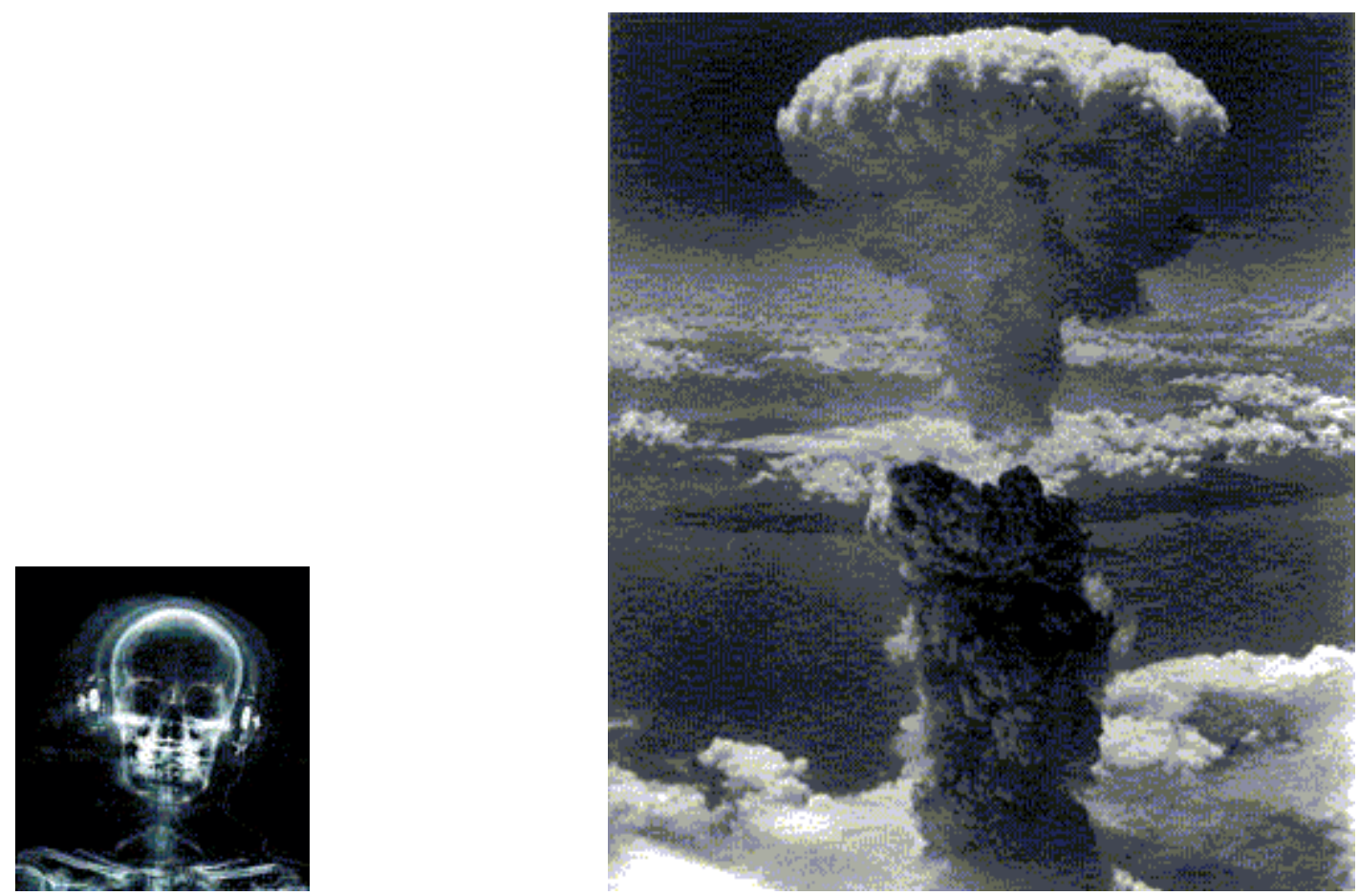

La Ciencia, en general, y la Física, en particular, han hecho posible una mejora apreciable de nuestra calidad y esperanza de vida. Desgraciadamente muchos descubrimientos científicos han sido utilizados para fines menos generosos.

Sin embargo, el campo de acción de la Física ha sido mucho más amplio en el pasado, de hecho fue llamada Filosofía Natural. Tal definición proviene de la propia etimología de la palabra, Physis = Naturaleza. A partir del siglo XIX la Física se restringió al estudio de los fenómenos físicos, dejando a otras Ciencias el estudio de esas otras leyes, no tan fundamentales, que parecen dominar nuestro entorno.

Pongamos un ejemplo. La Genética, como parte de la Biología, estudia las leyes y reglas de la herencia biológica, haciendo uso de métodos totalmente científicos. No obstante, sabemos que esas reglas se entenderían mejor si conociéramos las leyes y reglas que gobiernan las moléculas que componen los genes. Ese tipo de estudio lo realiza la Bioquímica, que, de una forma simple, se puede considerar parte de la Química. Pero es evidente que las interacciones entre las moléculas se podrían 
explicar estudiando a fondo las fuerzas eléctricas, magnéticas, gravitatorias y nucleares que actúan entre los componentes de dichas moléculas. Habríamos entrado de lleno en el campo de acción de diversas partes de la Física. A pesar de ello, lo enmarañado del fluir hereditario hace necesario el concurso de tres Ciencias.

Haciendo uso de estos conceptos se suele representar la Ciencia como un árbol, cuyas raíces profundas crecen en el campo de la Metafísica NOTA_2, siendo el fuerte y grueso tronco la Física y las frondosas ramas las otras Ciencias (Biología, Geología, Química, etc.). Nos quedará por asignar un papel a la Matemática. Bajo nuestro punto de vista, las Matemáticas constituyen el lenguaje por excelencia de la Ciencia, pues permite expresar, sin ambigüedades y de forma compacta, las diferentes reglas que gobiernan los fenómenos y sus relaciones, motivo de estudio de las diferentes Ciencias. NOTA_3

Para una mejor comprensión del estudio de la Física, ésta se suele estructurar en grandes ramas que consideran los distintos aspectos de la realidad, pero que están estrechamente relacionadas. Esta división, que en el pasado era más una exigencia de método, se ha convertido en necesidad hoy día, debido a la enorme complejidad de la Ciencia actual. Una división muy clásica sería: Mecánica (fuerzas y movimientos, cualquiera que sea su origen), Termología (fenómenos caloríficos), Electromagnetismo (fenómenos eléctricos y magnéticos), Ondulatoria (propagación de perturbaciones a grandes distancias), Óptica (particularización de la ondulatoria a los fenómenos luminosos), Física Atómica y Nuclear (estructura interna de la materia), Mecánica Cuántica (comportamiento microscópico de los fenómenos físicos). Como decíamos anteriormente, esta clasificación es muy clásica, y se pueden hacer otras atendiendo a según qué criterios. En cualquier caso, la especialización de los profesionales de la Física, debido a esa complejidad antes mencionada, hace posible aún subdivisiones dentro de cada una de las ramas enumeradas. 
A pesar de la complicado que parece el Cosmos, la Física no ha renunciado a su vocación simplificadora de la comprensión de la Naturaleza y sigue esforzándose por encontrar leyes y reglas, aún más fundamentales que las conocidas, que puedan explicar el conjunto de nuestro conocimiento. Hasta ahora sólo se conocen cuatro fuerzas fundamentales: Gravitatoria, Electromagnética, Nuclear Fuerte y Nuclear Débil. Una gran parte de los físicos contemporáneos siguen trabajando en lo que ha sido una aspiración de todos los físicos de la historia, encontrar una fuerza única que pueda explicar y contener a las cuatro anteriores como casos particulares, o como expresiones de ella bajo diferentes situaciones.

En resumen, la Física es la Ciencia experimental por excelencia, pues se nutre de la observación de la Naturaleza. Inclusive, los físicos puramente teóricos trabajan con experimentos, aunque éstos sean mentales, es decir, producto de la aplicación de las normas de la lógica a los fenómenos naturales. Los experimentos los puede suministrar directamente nuestro entorno, como en el caso de la Astrofísica, o se pueden diseñar y realizar en el laboratorio, como hace la Física de Altas Energías. En ambas situaciones, la pretensión del físico es la de elaborar primero un modelo de lo observado, para después elaborar una teoría, que contenga esas Leyes antes mencionadas y pueda predecir, con la máxima exactitud posible, la evolución de ese mismo fenómeno, cuando se cambia alguna de las circunstancias en las que se produce, además de prever nuevos fenómenos.

Consecuentemente, el físico modeliza la Naturaleza. Partiendo de la premisa fundamental de la inexistencia de una verdad absoluta nacida de la imperfecta observación del ser humano, sólo podemos reunir los datos proporcionados por ella, aquellos que somos capaces de observar para construir un modelo que dé cuenta de todos los aspectos y fenómenos detectados; al igual que un arquitecto o ingeniero construye una maqueta que da idea aproximada de lo que serán el edificio o la máquina una vez construidos. Si la observación es imperfecta, el modelo lo será aún 
más, pero la propia incertidumbre (ver la definición de incertidumbre más abajo) inherente a la cuantificación de los fenómenos observados, en el sentido clásico de medirlos y compararlos con otros, nos permite hacer modelos que se aproximan espectacularmente al mundo real percibido.

Los modelos se van perfeccionando y, por supuesto, cotejando con nuevos experimentos, bien idénticos a los que le dieron nacimiento, bien involucrando los fenómenos predichos por el modelo, para verificar su validez. El modelo se supone válido mientras no haya un experimento que demuestre lo contrario. De esta forma van descubriéndose nuevas leyes, complementarias de las que dieron lugar al modelo e, incluso, más simples y fundamentales, capaces de englobar a las primitivas. Paulatinamente, se va construyendo todo un cuerpo de información compuesto de hipótesis, teoremas, modelos, leyes y principios debidamente comprobados y verificados experimentalmente, más otras hipótesis, modelos, leyes, etc. por verificar. Este conjunto sintético y cerrado, que da cuenta de todos aquellos aspectos de la naturaleza que podemos interrelacionar en nuestra limitada capacidad constituye una teoría.

En unos casos, las teorías físicas se deducen de los experimentos, generalizando, y en otros se parte de una serie de hipótesis, que, al desarrollarse, generan una teoría cuya validez debe ser confirmada con la observación experimental.

A lo largo de los siglos, se ha ido perfilando, pues, la misión de la Física como la tarea de descubrir las leyes que gobiernan nuestro Universo. Pero la Física no puede pretender explicarlo todo, y menos aún con ecuaciones que describan cada uno de los procesos que se dan, se han dado o se darán en el futuro, en el Universo. La Física no puede pretender describir matemáticamente cada una de las interacciones entre cada una de las partículas que componen el Universo y el mundo que las rodea. Tal pretensión nos llevaría a una exposición tan complicada, que superaría en muchos 
grados la complejidad del propio Universo. ¿Qué sentido tendría, entonces, hacer física? Para eso ya tenemos el Universo en sí mismo, como un libro abierto que contiene toda la información posible. Sólo debemos aprender a leer el libro y esa es la misión de la Física, es decir, debe reducir el conocimiento a una forma más ordenada y satisfactoria, asequible a las limitaciones de la mente humana, pudiendo aplicarse a la solución de problemas de importancia práctica para la Humanidad.

En cualquier caso, el método físico nos lleva a cuantificar, es decir, a medir y valorar una serie de magnitudes que caracterizan los fenómenos estudiados en esos experimentos y que, a su vez, expresan la valoración de la validez del modelo, hipótesis o ley en discusión. Estas magnitudes, denominadas observables, son la expresión de lo que, en principio, podemos percibir con nuestros sentidos, si bien los físicos, a lo largo de la historia, se las han ingeniado para ampliar el rango de percepción de los sentidos, mediante la construcción de aparatos de medida, a veces tan sofisticados, que escapan de la comprensión del profano. Precisamente las relaciones entre esas magnitudes, usando las matemáticas como herramienta, permiten encontrar nuevas magnitudes observables y expresar las leyes en forma de ecuaciones o fórmulas, siendo entonces transmitidas y comprendidas de forma más rápida y concisa. Esas relaciones son las que permiten realizar predicciones y están en el origen del avance científico y tecnológico. Por ello, la Física ha sido también llamada Ciencia de las Medidas. Sobre este particular, en el siglo XIX, Lord Kelvin afirmó: "Cuando un científico puede medir algún fenómeno y expresarlo numéricamente, es cuando se puede decir que conoce algo acerca de dicho fenómeno. Cuando no lo puede medir o no es capaz de cuantificarlo, su conocimiento es escaso e insatisfactorio. En este caso podrá ser el comienzo del desarrollo de una teoría, pero no llega a ser exactamente Ciencia". 


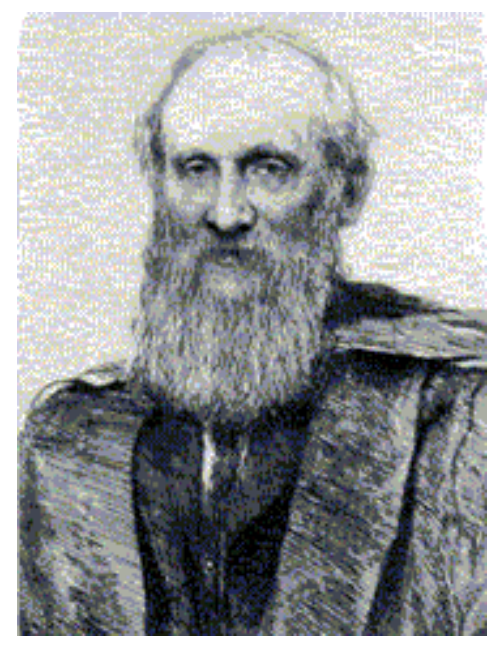

William Thomson, Primer barón de Kelvin

(Belfast, Irlanda, 26 de junio de 1824, † Netherhall en Largs, Escocia, 17 de diciembre, 1907)

Llegados a este punto, debemos hacer un esfuerzo por comprender que la imperfección de nuestros sentidos o de los aparatos de medida utilizados impiden absolutamente que las medidas realizadas sean exactamente coincidentes con los valores que se suelen llamar reales, los cuales nunca podremos encontrar o estar seguros de haber encontrado. ¿Qué sentido tiene hablar de valor real de una magnitud? El valor de una magnitud siempre estará sujeto a una medida en sí misma imprecisa, debido a imperfecciones del instrumento y debido a la incertidumbre causada por la interacción usada para medir, ambas imposibles de eliminar, por no hablar de las posibles influencias externas desconocidas que pueden estar presentes, por mucho que aislemos el sistema en experimentación. Eso sí, nos podemos aproximar con nuestras medidas a ese "valor real" cuanto mejores sean los aparatos y métodos utilizados, es decir, cuanto mejor sea su precisión, pero nada más. Hoy, tras los éxitos de la Teoría Cuántica, los físicos aceptan incluso la imposibilidad, por cuestión de principio, de alcanzar esos valores reales, debido a un hecho incuestionable: para poder observar hemos de interferir con el propio fenómeno observado y, consiguientemente, cambiamos las circunstancias que lo rodean y determinan su evolución en ausencia de nuestra observación. Como consecuencia lógica, los valores de nuestras medidas son inciertos, en mayor o menor cuantía dependiendo del tipo de experimento y herramientas utilizadas. Por ello, decimos 
que toda medida está afectada por una incertidumbre, de la cual los físicos han aprendido, a su vez, a cuantificar su valor máximo o mínimo. Note el lector que hablamos de incertidumbre y no de error, como era habitual en textos no tan antiguos. La diferencia es esencial. La incertidumbre es un hecho inherente a la propia observación y las circunstancias cambiantes que la rodean y que nosotros no podemos controlar y no a nuestra impericia o desconocimiento, lo cual constituiría un error.

Todo lo anterior llevó a Einstein a comparar la Física con una novela de aventuras e intriga. Es el propio lector quien debe descifrar el misterio por su cuenta, siguiendo una trama lógica que lleva, indefectiblemente, y en el momento esperado, al descubrimiento de la solución anunciada. El problema fundamental reside, no obstante, en que la mayor parte del misterio está por resolver, y no estamos seguros de la existencia de una solución, al menos usando el conocimiento y los métodos actuales. Afortunadamente pues, la Física está abierta a nuevos descubrimientos.

Por otra parte, como hemos mencionado más arriba, el Hombre, al examinar los experimentos e investigar su propio entorno, no puede hacer otra cosa que utilizar sus sentidos, a veces potenciados con instrumental sofisticado. Pero ello le conduce, infinidad de veces, a crearse "ilusiones". Por eso mismo, no podemos concebir la Física, ni cualquier otra ciencia experimental, como algo establecido e inmutable. La puerta ha de estar abierta a la crítica, la interpretación y el cambio. A veces, esos cambios resultarán de tal impacto y tan contrarios al sentido común, que costará aceptarlos, pero se debe desconfiar de lo aparentemente obvio. El propio Copérnico dice en las Revoluciones: "Las ideas aquí establecidas son muy difíciles, casi imposibles de aceptar, están en total contradicción con las creencias populares". A algunos científicos los cambios inaceptados les llevaron a la hoguera. 


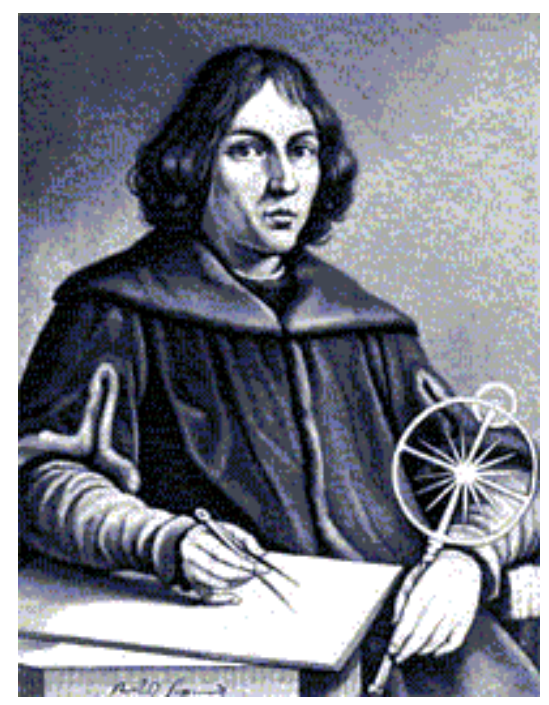

Nicolás Copérnico

(Torun, Polonia, 19 de febrero de 1473, † Frombork, Polonia, 24 de mayo de 1543)

Permítasenos un comentario final sobre un hecho incontestable. El Hombre tiene una tendencia morbosa a dogmatizar. Así, es práctica muy común defender los modelos y teorías elaborados como verdades absolutas e inmutables. Si el lector pregunta a un físico cuántico sobre la validez de la Mecánica Cuántica, estará expuesto a recibir una respuesta que no se diferencia prácticamente nada de los dogmas defendidos por muchas religiones.

\section{Notas}

NOTA 1: Sobre la distinción de leyes, teoremas y principios, ampliaremos los conceptos en un próximo artículo.

NOTA 2: La palabra Metafísica proviene del griego "meta ta physika", y significa "después de las cosas de la naturaleza", por consistir en una obra recopilatoria de tratados de Aristóteles, aparecida después de la Physika. Hoy definimos la metafísica como la investigación racional del ser en cuanto ser y de los principios más elevados del pensamiento y la existencia del Hombre y de todo lo que le rodea; en realidad, de todo lo relativo a lo que va más allá de la experiencia, sin recurrir a la fe, sólo utilizando la razón. 
Francisco Suárez, conocido como Doctor Eximius, sistematizó la Metafísica y la concibió como disciplina científica independiente, lo cual fue aceptado y utilizado por los grandes metafísicos de la historia: Descartes, Spinoza, Malebranche y Leibniz. Sin embargo, fue a partir del siglo XVIII cuando la Metafísica cobró su verdadera dimensión como ciencia de la explicación racional, frente a la ciencia empírica, es decir, experimental. Desde este punto de vista, toda especulación científica, desprovista de una labor experimental, se puede considerar Metafísica y supone la base racional de la Física.

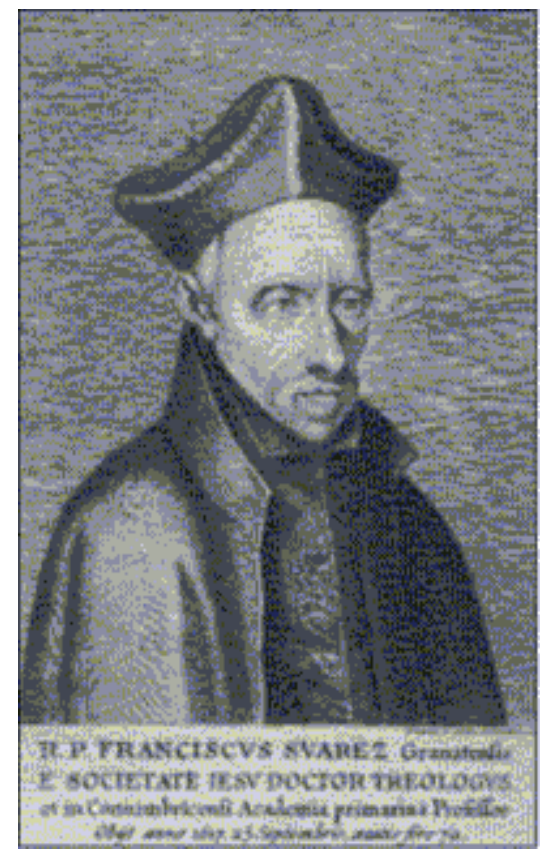

Francisco Suárez

(Granada, 5 de enero de 1548, † Lisboa, 25 de septiembre de 1617, teólogo, filósofo y jurista español) 


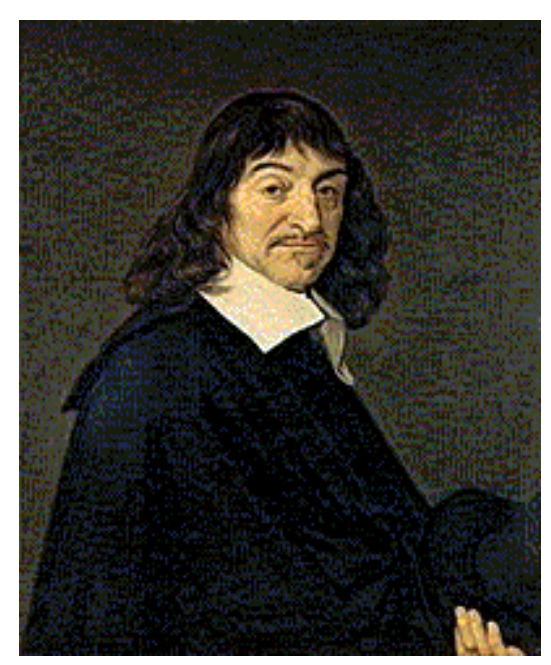

Réné Descartes

(La Haye, Touraine, cerca de Poitiers, 31 de marzo, 1596, † 11 de febrero, 1650)

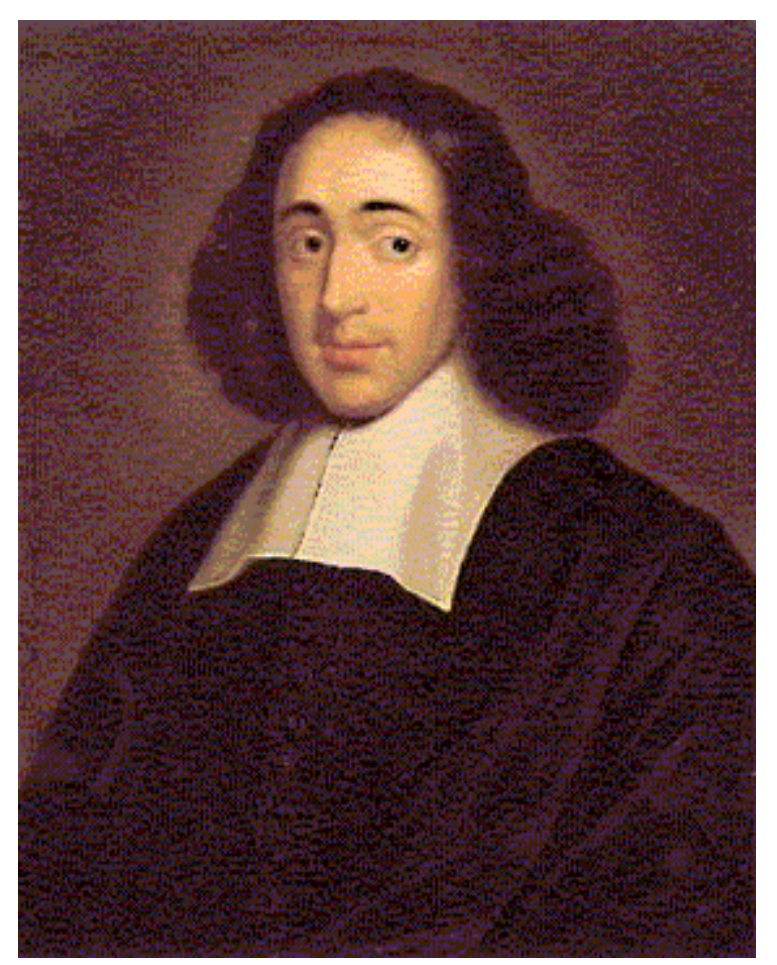

Baruch Spinoza

(Ámsterdam, 24 de noviembre de 1632, † La Haya, 21 de febrero de 1677) 


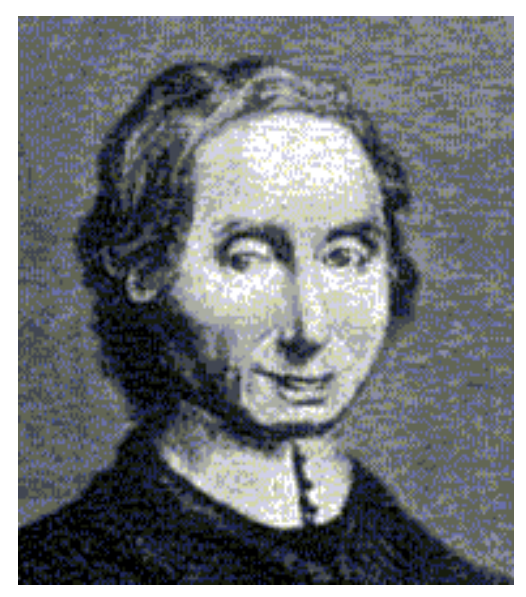

Nicolás Malebranche

(París, 1638, † 13 de octubre de 1715)

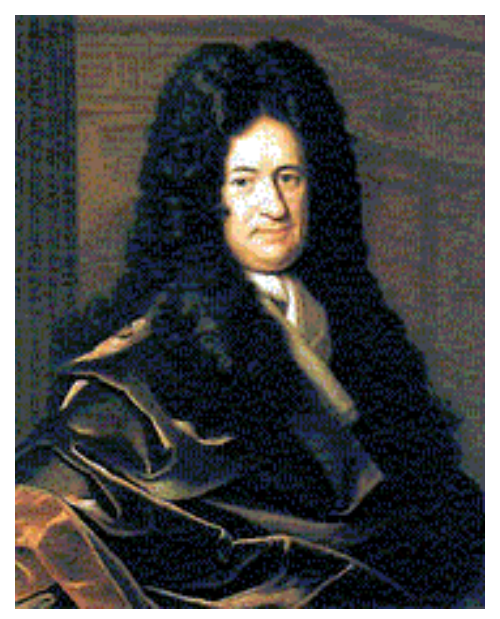

Gottfried Wilhelm von Leibniz

(Leipzig, 1 de julio, 1646, † 14 de noviembre, 1716)

Empero, durante el siglo XIX, la metafísica cayó en desgracia, debido al tremendo empuje de los éxitos de la Física como ciencia experimental. Los mecanicistas pensaron que, aplicando las leyes de Newton, serían capaces de explicarlo todo, hasta el nacimiento del Universo; no necesitaban de metafísica alguna. Ciertamente, había mucho por hacer, sobre todo descubrir el porqué de los principios físicos, pero eso no era labor de científicos; poco importaba si los principios quedaban en el misterio, era suficiente con observarlos experimentalmente y describirlos 
matemáticamente. Se pensaba en la Metafísica como una mera especulación de lo que es imposible conocer.

Pero el avance científico tomó otros rumbos. La complejidad de los nuevos fenómenos descubiertos a comienzos del siglo $X X$, la introducción del indeterminismo por la Mecánica Cuántica y la imposibilidad de construir una visión coherente del mundo físico con los simples resultados experimentales, indujeron a retomar la Metafísica como la raíz de toda ciencia, en otras palabras, como el método racional que pudiera arrojar alguna luz sobre aquello que la Física y las demás Ciencias positivas no conseguían explicar.

NOTA 3: Sobre este aspecto insistiremos en un futuro artículo, en el que hablaremos ampliamente de las relaciones entre la Física y las Matemáticas. 